\title{
Comparison of Three Electromechanical Oscillation Damping Estimation Methods
}

\author{
Jukka Turunen, Student Member, IEEE, Jerry Thambirajah, Student Member, IEEE, Mats Larsson, \\ Member, IEEE, Bikash C. Pal, SMIEEE, Nina F. Thornhill, SMIEEE, Liisa C. Haarla, SMIEEE, \\ William W. Hung, Alex M. Carter, and Tuomas Rauhala, Member, IEEE
}

\begin{abstract}
This paper describes three data driven methods to monitor electromechanical oscillations in interconnected power system operation. The objective is to compare and contrast the performance of the methods. The accuracy of damping ratio and frequency of oscillations are the measures of the performance of the algorithms. The advantages and disadvantages of various techniques and their limitations to measurement noise have been considered while assessing performance. The target frequency and damping are computed using the Nordic power system simulation model.
\end{abstract}

Index Terms-Independent component analysis, power system dynamic stability, power system monitoring, principal component analysis, state-space identification, wavelet transforms, Wide-Area Measurement System (WAMS).

\section{INTRODUCTION}

$\mathrm{E}^{\mathrm{L}}$ LECTROMECHANICAL oscillation is an inherent property of an ac transmission system and cannot be entirely eliminated. In many cases, the damping of inter-area electromechanical oscillation sets the limits to power transfer capacity [1]. In addition, the oscillations may pose a serious threat to system security if they are not controlled properly. From an operational point of view, it would be of high priority to be able to estimate the damping of the oscillations reliably in real-time in order to take appropriate and timely measures to keep the system stable.

Several methods estimate the damping from power system response to ambient excitations caused by e.g. variation in loads and generation [2]-[9]. References [3]-[9] present case studies where different methods are described and their performance is tested with measured and simulated data of a specific power system. Some methods have been implemented in commercial or non-commercial Wide-Area Measurement

J. Turunen and L. Haarla are with Aalto University School of Electrical Engineering, Espoo, Finland (e-mail: jukka.turunen@aalto.fi; liisa.haarla@aalto.fi).

J. Thambirajah, B. C. Pal, and N. F. Thornhill are with Imperial College London, London, UK (e-mail: jthambir@ic.ac.uk; b.pal@imperial.ac.uk; n.thornhill@imperial.ac.uk)

M. Larsson is with ABB Corporate Research, Baden, Switzerland (e-mail: mats.larsson@ch.abb.com).

W. W. Hung, and A. M. Carter are with National Grid, UK (e-mail: william.hung@uk.ngrid.com; alex.carter@uk.ngrid.com).

T. Rauhala is with Fingrid Oyj, Helsinki, Finland (e-mail: tuomas.rauhala@fingrid.fi).
Systems (WAMS) software and are in operational or experimental use in various countries [2], [10]-[16]. In addition, several methods were developed in the past in which it is attempted to estimate the damping from power system transient oscillations caused by e.g. major faults in the grid, loss of major generation or artificial probing signals [17]-[20].

The goal of the authors of this paper is to compare the performance of three methods for damping estimation intended for real-time use in WAMS systems such as the one described in [3]. The methods had independent origins and are tested on common data sets. The methods estimate the damping under the ambient conditions of the power system using the phasor measurements. The number of Phasor Measurement Units (PMU) and the quantities they are measuring are described in Sections VI B and VII B, respectively. Ambient data sets of simulated and measured grid data are studied. The paper is structured as follows. Section II classifies the damping observed in a power system and defines different measures of damping and relations between them. Section III describes the three damping estimation methods considered in this paper. Section IV describes the most important tuning parameters of the methods. Section V is a short description of the Nordic power system. Section VI reviews the data used to compare different damping estimation methods. Section VII presents the results of the comparison. Section VIII considers the assumption of Gaussian excitation of the oscillations. Section IX presents the conclusions of this paper and Section $\mathrm{X}$ points out future work areas.

\section{SYSTEM DAMPING CONCEPTS}

This section briefly outlines the concepts of system damping and gives definitions needed for the paper.

\section{A. Damping in a linear system}

Fig. 1 represents a power system as a cause-and-effect diagram with load demands exciting the generators to generate power that then flows through the transmission system. In the case of a single wide area electromechanical oscillation mode, the relationship between power flow, $y(t)$, and the unmeasured load demand, $u(t)$, is second order:

$$
\frac{d^{2} y}{d t^{2}}+2 \zeta \omega_{n} \frac{d y}{d t}+\omega_{n}^{2} y(t)=u(t)
$$


where $\zeta$ is the damping ratio and $\omega_{n}$ is the natural frequency of the oscillation. If $\zeta$ is positive and less than 1 , an impulsive change in $u(t)$ gives a decaying transient oscillating response such as that shown in Fig. 2. This response typically occurs after a fault or major system disturbance and is called a transient or ring down. On the other hand, random variation in $u(t)$ excites a persistent oscillation at the natural frequency, which may be of small amplitude and hidden in noise. An example is presented in Fig. 5. This is called ambient operation. The methods presented in this paper aim to assess the stability of inter-area modes during ambient operation.

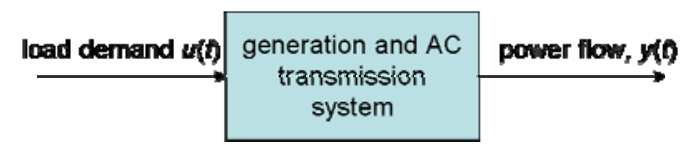

Fig. 1. Cause-and-effect diagram for ac transmission system.

\section{B. Impulse response}

When the input $u(t)$ is a unit impulse and $0<\zeta<1$ then (1) has the following solution which is illustrated in Fig. 2:

$$
y(t)=\frac{\omega_{n}}{\sqrt{1-\zeta^{2}}} e^{-\zeta \omega_{n} t} \sin \left(\omega_{n} \sqrt{1-\zeta^{2}} t\right)
$$

Equation (2) shows that the amplitude of the transient signal decays exponentially with a time constant $\tau$ which depends on the damping ratio and natural frequency, where $\tau=1 / \zeta \omega_{n}$. The frequency of oscillation $\omega_{t}$ is not the same as the natural frequency $\omega_{n}$, although the two are close if the damping ratio is small:

$$
\omega_{n}=\frac{\omega_{t}}{\sqrt{1-\zeta^{2}}}
$$

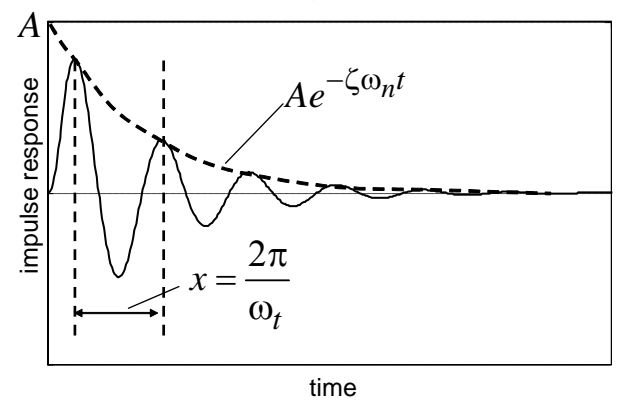

Fig. 2. Second order impulse response.

\section{Damping ratio calculations}

Logarithmic Decrement: If an impulse response curve is available then the damping ratio may be determined via the logarithmic decrement $\delta=\ln (y(t+x) / y(t))$, where $y(t)$ and $y(t+x)$ are the magnitudes of successive peaks. From (1), $y(t+x)=y(t) e^{-\zeta \omega_{n} x}$ where $x=2 \pi / \omega_{t}$ is the time interval between successive peaks. Rearrangement and substitution of $\omega_{n}=\frac{\omega_{t}}{\sqrt{1-\zeta^{2}}}$ leads to $\zeta=\frac{\delta}{\sqrt{(2 \pi)^{2}+\delta^{2}}}$. Damping ratio may be expressed as a percentage, hence $\zeta=0.1$ would be expressed as $10 \%$ damping. The impulse response curve is not directly available in ambient operation, however it can be estimated from the ambient output as described in Sections III $\mathrm{B}$ and III C.
Eigenvalue analysis: Eigenvalues are the complex roots, $\lambda_{1}$ and $\lambda_{2}$, of the characteristic equation arising during the solution of the second order differential equation:

$$
\begin{aligned}
& \lambda^{2}+2 \zeta \omega_{n} \lambda+\omega_{n}^{2}=0 \\
& \lambda_{1,2}=-\zeta \omega_{n} \pm j \omega_{n} \sqrt{1-\zeta^{2}}
\end{aligned}
$$

Eigenvalues are also called system poles. There are many ways to estimate eigenvalues from time series of measurements $y(t)$, including subspace identification which is described later. If the eigenvalue is expressed in Cartesian form as $\lambda_{i}=\alpha \pm j \omega_{t}$, then the magnitude of the real part $\alpha=\zeta \omega_{n}$ is called the attenuation while the imaginary part $\omega_{t}=\omega_{n} \sqrt{1-\zeta^{2}}$ is the frequency of oscillation. An expression for damping ratio is as follows:

$$
\zeta=\frac{-\alpha}{\sqrt{\alpha^{2}+\omega_{t}^{2}}}
$$

Damping is positive if $\alpha$ is negative. Often the system has multiple eigenvalues, however for a dominant inter-area mode, the complex conjugate eigenvalues whose imaginary parts correspond to the observed mode frequency would be used in the damping estimation.

Other measures of damping: Some alternative ways of specifying damping exist, as follows:

- Time constant, $\tau$, and decay time: The time constant $\tau$ is the time taken for the amplitude of the oscillation to decay to $1 / \mathrm{e}$ of its original value. Decay time is typically defined as $4 \tau$. The amplitude of the transient signal in (2) decays to less than $2 \%$ of its initial value within four time constants.

- Number of cycles before settling: Time constant and decay time do not take the frequency of the oscillation into account. A related measure which does take account of the frequency is the ratio between the decay time and the period of oscillation, i.e. the number of cycles of oscillations occurring during a period of time equal to four time constants.

- Decay ratio: It is the ratio of magnitudes of subsequent peaks in the impulse response.

The above are different ways of describing the impulse response of Fig. 2. They can all be derived as the functions of $\alpha$ and $\omega_{t}$ hence do not add any new information. The reason for mentioning them is that commercial system monitoring tools may report one or more of these measures.

\section{DAmping Estimation Methods}

The damping estimation methods considered in this paper are based on (i) state-space system identification, (ii) spectral independent component analysis and random decrement, and (iii) wavelet transform and random decrement. Each of these methods is summarized below with relevant references given for more detailed explanations.

\section{A. Damping estimation based on subspace identification [21]}

The method is based on a general state space description of the power system dynamics in the so-called innovation form 


$$
\begin{aligned}
& x_{k+1}=A x_{k}+B u_{k}+K e_{k} \\
& y_{k}=C x_{k}+D u_{k}
\end{aligned}
$$

where the system matrices $A, B, C$, and $D$ describe the system dynamics and $K$ describes the way the noise affects the system. The vectors $x_{k}$ and $y_{k}$ model the internal state and the measured outputs of the model, at time instant $k$. The vector $u_{k}$ can be used to model external probing signal inputs that are applied to assist the identification of the system dynamics. This model is an ideal representation to use in a multivariable approach, and can potentially also be used for bias-free estimation in presence of non-white background load variations [21]. The system identification problem related to the form (6) typically consists of finding estimates of the system order and the system matrices. The discrete-time innovation form model can be converted to a corresponding continuous-time and then transformed using a modal decomposition [21] yielding

$$
\begin{aligned}
& \dot{\zeta}=\Lambda \zeta_{k}+\Phi^{-1} u_{k} \\
& y_{k}=C \Phi_{k} \zeta_{k}+D u_{k}
\end{aligned}
$$

The modal transformation decouples the modes from each other and the properties of each mode $i$ can be determined from the system matrix element $\Lambda=\operatorname{diag}\left(\lambda_{1} \ldots \lambda_{n}\right)$. From the eigenvalue $\lambda_{i}=\alpha_{i}+\mathrm{j} \omega_{i}$, related to mode $i$, the corresponding modal frequency $\omega_{i}$ and its damping ratio $\zeta_{i}$ can easily be determined.

Furthermore, the modal observability matrix is given by

$$
O=C \Phi
$$

whose elements $O_{(i, j)}$ describe how the mode $j$ is observable from the output $i$. The magnitude of the complex elements shows how observable a modal activity is in a particular output and the complex argument the phase of that activity. Mode shape analysis can be used to distinguish local modes from inter-area modes, and to identify which parts of the system swing in a coherent way. In this paper the subspace method MOESP is used to identify the system model [21]. The theoretical background and its relation to the N4SID method used in [4] and [7] is reviewed in detail in [22]. Compared to the ARMAX method used in [4], the main advantage of the subspace methods is that they provide a robust framework for model order estimation without the need for repeated system identification. This is a significant advantage for real-time applications since it reduces the computational complexity of the system identification.

\section{B. Damping estimation based on spectral independent component analysis and random decrement [23]}

This method consists of two distinct steps: (i) a modeselection step for the detection of the presence of inter-area modes and estimation of their frequencies via a multivariate analysis technique known as spectral Independent Component Analysis (spectral ICA) [24], and (ii) the estimation of the mode damping by estimating the system response via a technique known as Random Decrement (RD) [25].

Spectral ICA is a multivariate technique that decomposes a matrix of frequency spectra into common and independent non-Gaussian sources such that $\mathbf{X}=\mathbf{C D}$, where the rows of $\mathbf{X}$ are the spectra of different measured outputs realized as a linear superposition of maximally independent sources (referred to as independent components - ICs) in $\mathbf{D}$, in the specific ratios (referred to as significance indices - SIs) contained in $\mathbf{C}$. The ICs in $\mathbf{D}$ are narrowband spectra with single spectral frequency peaks corresponding to the inter-area modes in the data while the SIs in the columns of $\mathbf{C}$ are a measure of the relative strengths of the modes in each measured output. These SIs are normalized and have magnitudes bound between " 0 " and " 1 " such that the row with an SI of " 1 " corresponding to a certain IC (mode) represents the output in which the mode is strongest relative to the other outputs.

Using the information of the system modes obtained using spectral ICA, the signals are filtered, after which the RD method is applied. The RD method is a univariate technique that can be used to estimate the system response. It is an averaging technique in the time domain, analogous to the Welch Periodogram method in the frequency domain. The averaging technique yields a trend known as the Random Decrement (RD) signature, which is an estimation of the correlation function of a Gaussian process. In order to extract the RD signature from the ambient response, the RD method uses a threshold, $h$, so that every time, $t_{r}$, when the (mean centered) signal, $y$, crosses the threshold, a sample of the signal of length $\tau, y_{0}\left(t_{r}: t_{r}+\tau\right)$, is collected. The $N$ samples collected in a specific time window are averaged yielding the RD auto signature $R D_{Y Y}(\tau)$.

$$
R D_{Y Y}(\tau)=\frac{1}{N} \sum_{r=1}^{N} y\left(t_{r}: t_{r}+\tau\right)
$$

Under the assumption that the power system is linear and excited with Gaussian distributed random variations, the RD auto signature is proportional to the free decay or impulse response of the system. By fitting a second order decay function to the RD signature, the natural frequency and damping of each mode can be obtained. This univariate approach can be extended into a multivariate approach by combining the damping values obtained from each output for each mode using the SIs from spectral ICA. This serves the additional purpose of mitigating the effect of using a single output with poor modal participation. Using the SIs, the estimated system damping of the $s$ th mode, $\zeta_{s}$, can therefore be expressed by the following equation, where $m$ represents the number of measured outputs and $\zeta_{i}$ and $S I_{i}$ represent the damping estimation and significance index of the particular (sth) mode in the ith measured output:

$$
\zeta_{S}=\left(\sum_{i=1}^{m} \zeta_{i} S I_{i} / \sum_{i=1}^{m} S I_{i}\right)
$$

A schematic of the implemented algorithm (RD-ICA) is shown in Fig. 1. Complete details of the method are available in [23]. 


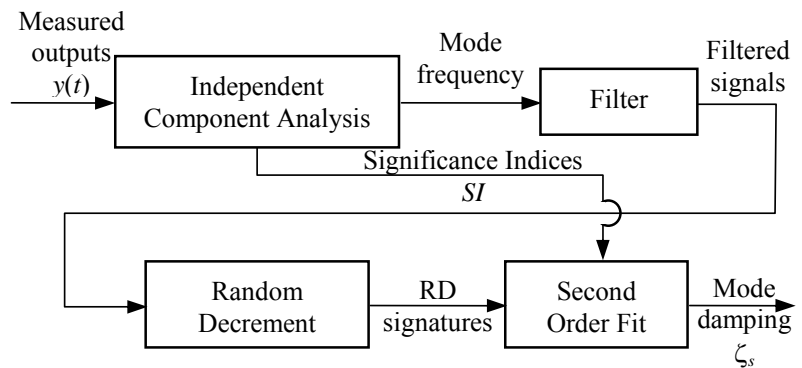

Fig. 3. Implementation of RD-ICA algorithm.

\section{Damping estimation based on wavelet transform and random decrement [26][27]}

The third method is a univariate method and it is based on the Continuous Wavelet Transform (CWT) and Random Decrement (RD) and it is schematically presented in Fig. 4. The wavelet transform is an effective method of extracting information of a signal in both the time and frequency domain [28]. The CWT of a signal $y(t)$ is calculated by computing the wavelet coefficients $C(a, b)$ at different scales $a$ and positions $b$ :

$$
C(a, b)=\int_{-\infty}^{\infty} y(t) \frac{1}{\sqrt{a}} \psi^{*}\left(\frac{t-b}{a}\right) d t
$$

where $\psi$ is a real wavelet function in case of the real CWT and a complex wavelet function in case of the complex CWT.

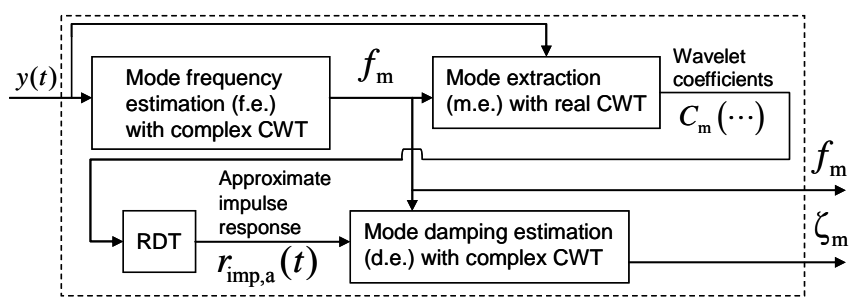

Fig. 4. Block diagram of the damping estimation method based on wavelet transform and random decrement. CWT is continuous wavelet transform and RDT is random decrement technique. Input $y(t)$ is the analyzed signal, and outputs $f_{\mathrm{m}}$ and $\zeta_{\mathrm{m}}$ are the estimated mode frequency and damping, respectively.

Mode frequency is estimated in the specified frequency band $(0.2-0.4 \mathrm{~Hz}$ in this case) with the complex CWT. The wavelet scale, $a$, that produces the highest average modulus of the (complex) wavelet coefficient (comparable to oscillation amplitude), is selected as the scale of the mode, $a_{\mathrm{m}}\left(\psi_{\text {f.e. }}\right)$. The scale is then converted to the frequency of the mode, $f_{\mathrm{m}}$, with the equation

$$
f_{\mathrm{m}}=\frac{f_{\mathrm{c}}\left(\psi_{\text {f.e. }}\right)}{a_{\mathrm{m}}\left(\psi_{\text {f.e. }}\right) \cdot \Delta}
$$

where $f_{\mathrm{c}}\left(\psi_{\mathrm{f} \text {.e. }}\right)$ is the center frequency of the frequency estimation wavelet function, $\psi_{\text {f.e., }}$ and $\Delta$ is the signal sampling period.

After knowing the mode frequency, $f_{\mathrm{m}}$, the mode is extracted from $y(t)$ with the real CWT by calculating the resulting wavelet coefficients with (11). The parameter $a_{\mathrm{m}}\left(\psi_{\text {m.e. }}\right)$ is used as the wavelet scale, $a$, corresponding to the estimated mode frequency, $f_{\mathrm{m}}$, according to the equation

$$
a_{\mathrm{m}}(\psi)=\frac{f_{\mathrm{c}}(\psi)}{f_{\mathrm{m}} \cdot \Delta}
$$

where $f_{\mathrm{c}}(\psi)$ is the center frequency of the mode extraction wavelet function, $\psi_{\text {m.e. }}$. The resulting wavelet coefficients are (approximately) linearly dependent on the instantaneous value of the oscillation mode at different time instances, $b$ [28]. Therefore the mode damping information is (approximately) preserved during the mode extraction.

After extracting the mode of interest from the signal, $y(t)$, the RD method is applied to convert the single-mode ambient response to approximate impulse response (or RD signature) from which the damping can be estimated. The RD method is described in Section III B.

Mode damping is received from the approximate impulse response by calculating at first the complex wavelet coefficients, $C_{\text {imp }}(\cdots)$, at different time instances, $b$, with (11). The parameter $a_{\mathrm{m}}\left(\psi_{\text {d.e. }}\right)$ is used as the wavelet scale, $a$, corresponding to the estimated mode frequency, $f_{\mathrm{m}}$, according to (13) where $\psi_{\text {d.e. }}$ is the wavelet function used in the damping estimation from the approximate impulse response.

The damping ratio of the mode, $\zeta_{\mathrm{m}}$, is finally calculated by using wavelet coefficients, $C_{\text {imp }}(\cdots)$, from two different time instances:

$$
\zeta_{\mathrm{m}}=-\frac{100}{2 \cdot \pi \cdot f_{\mathrm{m}} \cdot T_{\mathrm{d}}} \ln \frac{\left|C_{\mathrm{imp}}\left(T_{\mathrm{sp}}+T_{\mathrm{d}} / 2\right)\right|}{\left|C_{\text {imp }}\left(T_{\mathrm{sp}}-T_{\mathrm{d}} / 2\right)\right|}
$$

where $T_{\mathrm{d}}$ is the difference between the positions (or time instants), $b$, of the wavelet coefficients, $C_{\text {imp }}(\cdots)$, in the damping calculation, and $T_{\mathrm{sp}}$ is the time instant from the beginning of the approximate impulse response, needed for the damping calculation. Complete details of the method, including the selection of parameters and wavelet types, are available in [26] and [27].

\section{Tuning Parameters of THE Methods}

When the methods are applied to analyze power system data, they require some tuning. The most important tuning parameter for the three methods is the length of the sliding time window. In the RD-ICA and wavelet methods also the threshold for averaging needs to be tuned as well as the length of RD signature. These parameters can however be tuned with relation to the frequency range of detection. In [23] it is described how the optimal values for the parameters were obtained for the inter-area mode range of detection. The RD method allows the automated selection of tuning parameters in between the mode estimation and damping estimation steps for all frequency ranges. This allows the method to be used easily when multiple modes are present in the data without the need of prior information about the modes. In the wavelet method, the frequency band of the frequency estimation needs to be adjusted to include the mode of interest.

\section{Characteristics OF THE STUdy System}

The study system considered in this paper is the synchronous Nordic power system consisting of the grids of 
Finland, Sweden, Norway and Eastern Denmark. There are several HVDC connections to neighboring synchronous systems [16].

There are two main inter-area oscillation modes in the system. Those are at about $0.3 \mathrm{~Hz}$ and at about $0.5 \mathrm{~Hz}$. In the $0.3 \mathrm{~Hz}$ mode, the generators in southern Finland oscillate against the generators in Southern Sweden and Norway. In the $0.5 \mathrm{~Hz}$ mode, the generators in Southern Norway and Southern Finland oscillate against the generators in Southern Sweden [29]. Oscillations of the $0.3 \mathrm{~Hz}$ mode arise typically when power is transferred from Southern Finland to Southern Sweden via interconnecting ac lines at North, and damping of the oscillations sets the limit to power transfer capacity in that direction. The focus of this paper is mainly on the $0.3 \mathrm{~Hz}$ mode oscillation. Section VII C also examines the ability of the methods to deal with the second simultaneously present $0.5 \mathrm{~Hz}$ mode.

\section{REVIEW OF THE DATA USED IN COMPARISON OF THE DAMPING ESTIMATION METHODS}

Both simulated data and measured grid data are used in comparison of the methods.

\section{A. Simulated Data}

A detailed simulation model of the Nordic power system is used in the study. It has about 6000 buses, 1700 machines and 2600 loads. The number of state variables is about 17000 .

The power flow case in the simulation is tuned in such a way that the power transfer through the two $400 \mathrm{kV}$ ac lines from Finland to Sweden approximately matches with the measured PMU data set. The power flow is about $1000 \mathrm{MW}$ in this case. This similarity enables the comparison between the $0.3 \mathrm{~Hz}$ mode damping estimates from the simulated data and from the measured PMU data. The simulation model is assumed to reflect well the actual system behavior and the interconnecting power flow is the dominant factor affecting the $0.3 \mathrm{~Hz}$ mode damping. The actual or reference smallsignal damping of the $0.3 \mathrm{~Hz}$ mode is in this case about $7 \%$. The reference damping is calculated by simulating a small transient and applying Prony analysis to find out the oscillation modes and their damping [4]. Because the simulated transient is small, the power system behavior is assumed to be linear and the damping derived can be considered as representing the small-signal damping of the power system.

To reflect the actual system behavior under ambient conditions, randomly varying loads excite the oscillations. The biggest load from each area of the Southern part of Finland is randomly varied. At these locations the variations excite the $0.3 \mathrm{~Hz}$ and the $0.5 \mathrm{~Hz}$ oscillations [29]. The individual loads follow the uniform distribution with a fixed margin. The load variation results in the driving noise being close to Gaussian. The simulated oscillation amplitudes become close to measured oscillation amplitudes in the grid.

\section{B. Measured PMU Data}

A data set of measured PMU data is analyzed in this paper.
The PMU measurements are from the nine PMUs in Finland located at the main interconnections, near the largest generators, and near the HVDC terminals. In the data set, power flow from Finland to Sweden varies, Fig. 5, and that should affect the damping estimates of the $0.3 \mathrm{~Hz}$ mode.

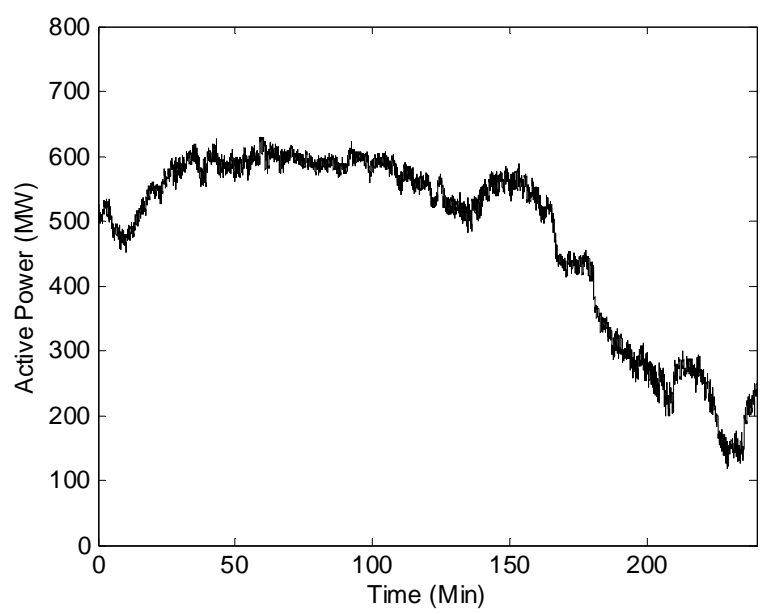

Fig. 5. Active power flow of an ac line between Finland and Sweden.

\section{Performance of the Damping Estimation Methods}

\section{A. Simulated Data}

Damping and frequency estimation results achieved by analyzing simulated data with the three described methods are presented in Table 1 and Table 2. The analyzing time window length is 11 minutes and the signal-to-noise ratio (SNR) of the analyzed signals is 5 with linear scale:

$$
S N R=\frac{P_{\text {signal }}}{P_{\text {noise }}}=\left(\frac{\sigma_{\text {signal }}}{\sigma_{\text {noise }}}\right)^{2}
$$

where $P_{\text {signal }}$ and $P_{\text {noise }}$ are the signal power and noise power, respectively, and $\sigma_{\text {signal }}$ and $\sigma_{\text {noise }}$ are the signal standard deviation and noise standard deviation, respectively. SNR of 5 is selected because it is a realistic value in case of the measured quantities analyzed in this paper [21].

The results for the simulated data in Table 1 and Table 2 indicate a high degree of consistency across the different methods. The effect of using different quantities for the estimation is also small. With the wavelet method, slightly lower mean values for the damping estimates are generally achieved. However, the estimated standard deviations of the damping ratio estimates account well for the differences in the mean damping ratios of the different estimation methods. The mean damping estimates obtained using the three methods are slightly lower than the $7 \%$ reference value. However, once again, considering the standard deviations of the estimates, the expected value nearly falls within the $67 \%$ confidence interval of the estimates. The frequency estimates are on the other hand more consistent across all methods and only slightly over the expected $0.3 \mathrm{~Hz}$ value. 
Table 1. Damping estimation results with simulated data. Time window length is $\mathbf{1 1}$ minutes, signal-to-noise ratio is 5 . The values in the last row correspond to the rightmost spots in Fig. 6 and Fig. 7.

\begin{tabular}{|l|l|l|l|}
\hline & $\begin{array}{l}\text { Damping estimate with } \pm \text { one standard deviation } \\
\text { error bounds }\end{array}$ \\
\hline \hline $\begin{array}{l}\text { Output } \\
\text { quantity }\end{array}$ & Subspace & RD-ICA & Wavelet \\
\hline \hline Grid & $0.0639 \pm$ & $0.0657 \pm$ & $0.0601 \pm$ \\
Frequencies & 0.0049 & 0.0057 & 0.0042 \\
\hline Bus Voltage & $0.0687 \pm$ & $0.0647 \pm$ & $0.0598 \pm$ \\
Magnitudes & 0.0048 & 0.0061 & 0.0053 \\
\hline Bus Voltage & $\mathbf{0 . 0 6 8 4} \pm$ & $\mathbf{0 . 0 6 3 5} \pm$ & $\mathbf{0 . 0 6 2 6} \pm$ \\
Angle & $\mathbf{0 . 0 0 3 7}$ & $\mathbf{0 . 0 0 6 5}$ & $\mathbf{0 . 0 0 4 7}$ \\
Differences & & & \\
\hline
\end{tabular}

Table 2. Frequency estimation results with simulated data. Time window length is 11 minutes, signal-to-noise ratio is $\mathbf{5}$ (linear scale).

\begin{tabular}{|l|l|l|l|}
\hline & \multicolumn{2}{|l|}{$\begin{array}{l}\text { Frequency estimate with } \pm \text { one standard deviation } \\
\text { error bounds }\end{array}$} \\
\hline \hline $\begin{array}{l}\text { Output } \\
\text { quantity }\end{array}$ & Subspace & RD-ICA & Wavelet \\
\hline \hline Grid & $0.3105 \pm$ & $0.3110 \pm$ & $0.3109 \pm$ \\
Frequencies & 0.0030 & 0.0063 & 0.0020 \\
\hline Bus Voltage & $0.3103 \pm$ & $0.3111 \pm$ & $0.3114 \pm$ \\
Magnitudes & 0.0027 & 0.0034 & 0.0019 \\
\hline Bus Voltage & $0.3070 \pm$ & $0.3101 \pm$ & $0.3040 \pm$ \\
Angle & 0.0023 & 0.0041 & 0.0025 \\
Differences & & \multicolumn{2}{l}{} \\
\hline
\end{tabular}

The effect of analyzing window length on the mean values and standard deviations of the damping estimates in case of different methods are presented in Fig. 6 and Fig. 7, respectively. In general, the mean values of the damping estimates increase and approach the real damping of the mode (about 7\%) as the time window length increases. The subspace method, instead, shows slightly less bias than the RD-ICA and wavelet methods for all tested time window lengths.

The RD-ICA method has the lowest damping estimate standard deviation with the shortest time windows and the subspace method with the longest time windows. The effect of analyzing time window length on the damping estimate standard deviations is greatest for the wavelet method. With the longest time windows, the standard deviations for wavelet method are comparable to other methods. The higher values of the standard deviations of the estimates for the wavelet method are attributable to the univariate nature of the method. The wavelet method requires the selection of a suitable signal for analysis and therefore is subject to variations in the estimate with respect to the optimality of the selection. The RD-ICA and subspace methods on the other hand are multivariate in nature and are therefore less sensitive to this criterion since all signals are used in the estimation. The results however suggest that a window length of at least 7 minutes is necessary for all methods in order to minimize the standard deviation of the damping estimates.

The effect of measurement noise on the mean values of the damping estimates for the different methods is presented in Fig. 8. It can be concluded that measurement noise in the studied range has a negligible effect on the estimates for all the studied methods. In the studied cases, the standard deviations remain unaffected, too. In [21] it is shown that the dominant mode can be estimated reliably until the SNR of 1, and the estimation of other mode is reliable when the SNR is 5 or more. Measurement noise here refers to uncertainty in the PMU measurement, and not to the noise in the transmission grid due to random load variations.

In all cases, the small differences between the expected and estimated results may be attributed to the nature of the simulation and its corresponding effect on the simulated data. Since small deviations of the loads were made at certain chosen locations drawn from a uniform distribution and a constant period of application, the resulting data that is analyzed is theoretically not Gaussian in nature. In such circumstances the RD-ICA and wavelet methods are expected to underperform, because the assumptions made in the RD method specifically require Gaussian excitation in order to make a correct estimation of the system impulse response. The subspace method on the other hand is less affected and therefore yields results with a lower bias.

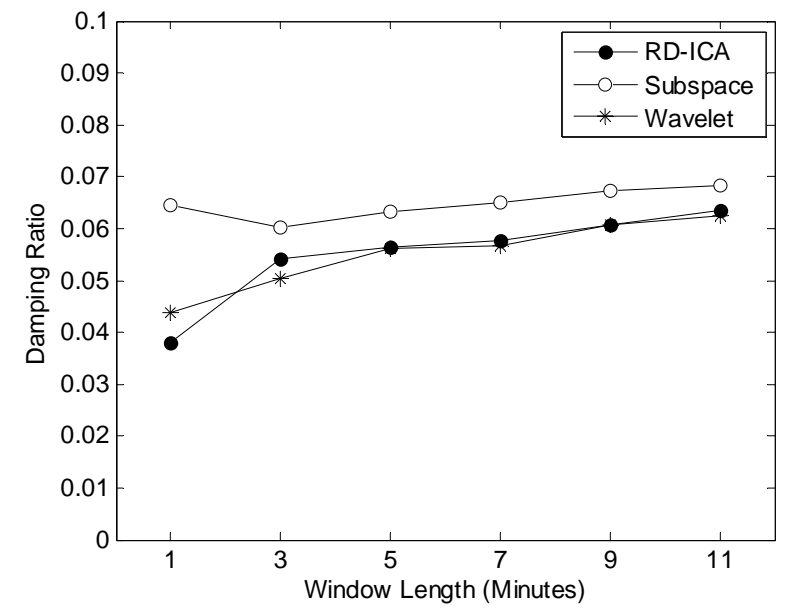

Fig. 6. Damping ratio as a function of analyzing time window length. The studied input quantities are the simulated bus voltage angle differences.

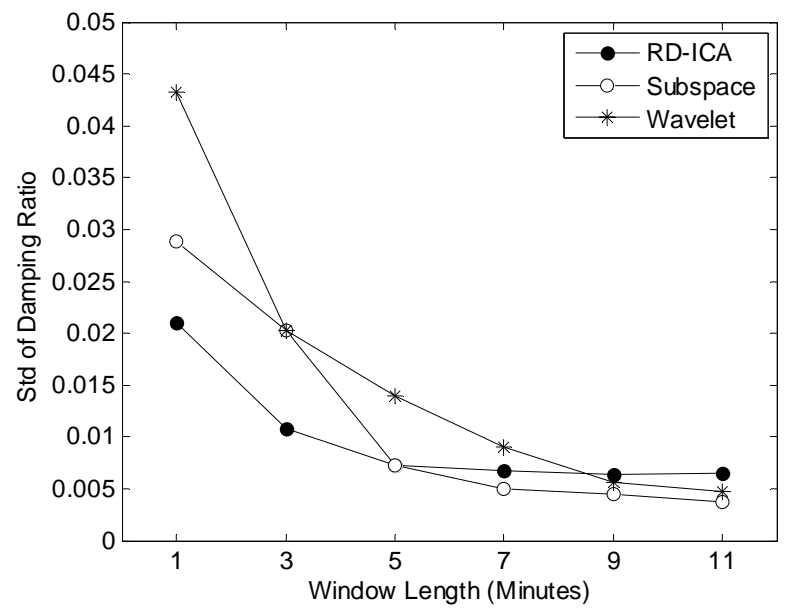

Fig. 7. Standard deviations (std) of damping ratios as a function of analyzing time window length. The studied input quantities are the simulated bus voltage angle differences. 


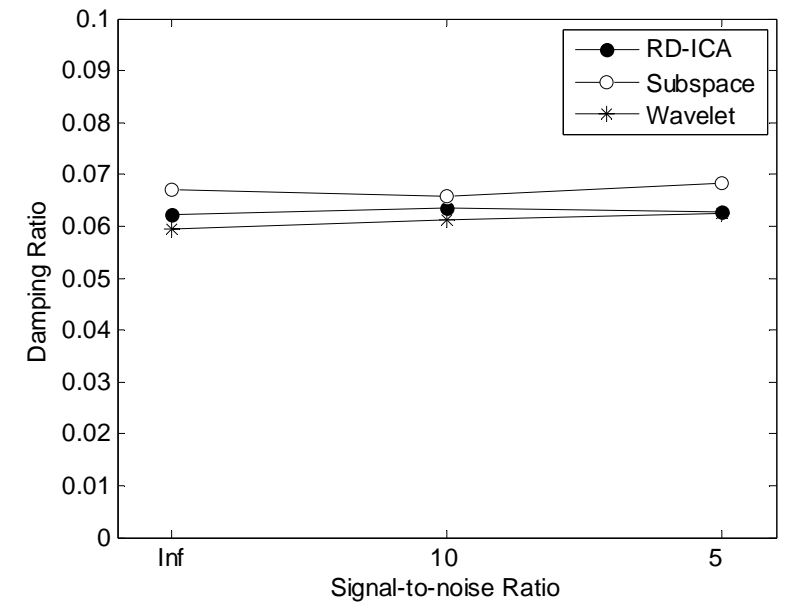

Fig. 8. Damping ratios as a function of signal-to-noise ratios of the analyzed signals. The studied input quantities are the simulated bus voltage angle differences.

\section{B. Measured Data}

Damping and frequency estimation results achieved by analyzing measured PMU data using the three methods are presented in this section. The episode studied is the one shown in Fig. 5 but also here several quantities are analyzed: system frequencies, the power flows of Finland-Sweden interconnection, and voltage angle differences. The processing of the measurements is described in case of each method in Section III.

The results of the damping estimation for the subspace, RDICA, and wavelet methods are presented in Fig. 9, Fig. 10, and Fig. 11, respectively. The analyzing time window length is 10 minutes. From the results, it can first be observed that the damping estimates are consistent for the different input quantities in each particular method. There is also a variation in the damping estimate over the time of the estimation for all methods. This variation exists because of fluctuations in the levels of power transfer over the period of estimation. Additionally, changes in loads and generation can lead to a change in damping levels. Filtering helps smooth out the results.

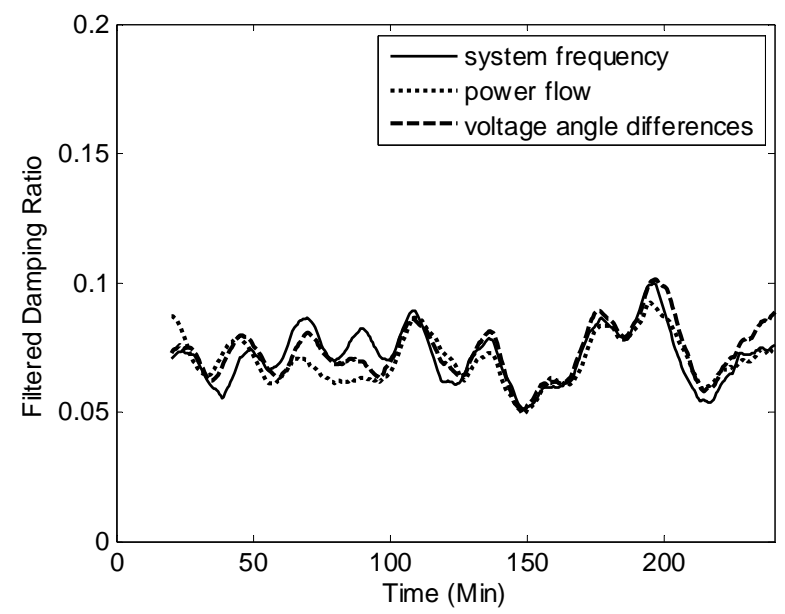

Fig. 9. Filtered damping ratio as a function of time for the subspace method The studied input quantities are indicated in the legend.

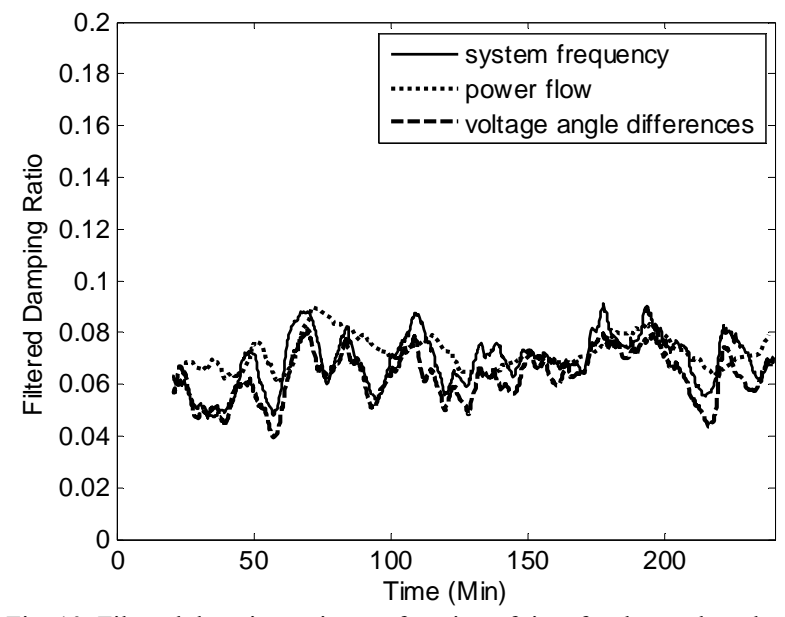

Fig. 10. Filtered damping ratio as a function of time for the random decrement method. The studied input quantities are indicated in the legend.

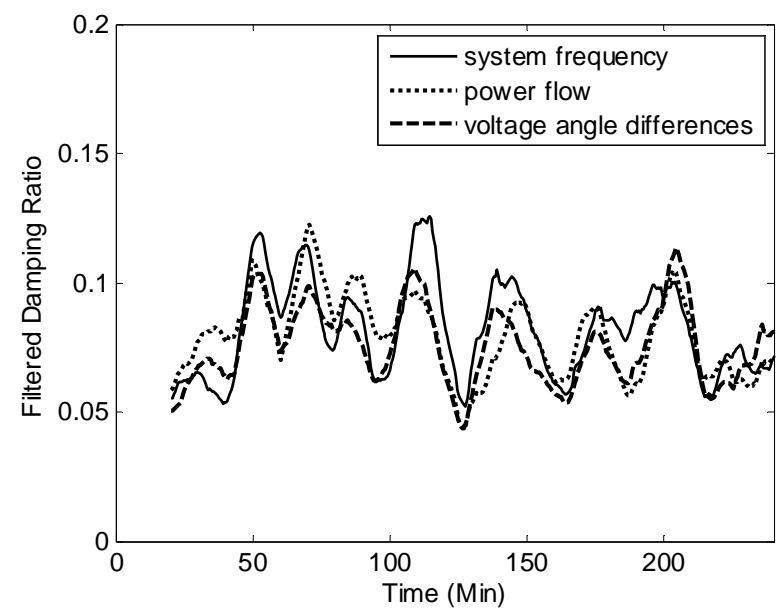

Fig. 11. Filtered damping ratio as a function of time for the wavelet method. The studied input quantities are indicated in the legend.

Comparisons of the frequency and damping estimation results are presented in Fig. 12 and Fig. 13, respectively. The results indicate that the frequency estimates have less variance than the damping estimates. They are additionally consistent across all the studied methods. The variations in the damping estimates, that were initially observed in the individual method results of Fig. 9, Fig. 10, and Fig. 11, coincide quite well when the results are compared. However the level of bias differs during different time intervals. The intervals where the estimations coincide are episodes when the mode energy in the signals is high allowing for better estimation of the damping than at other times. This is indicated by the results that show this estimate to be between $6-8 \%$ at such times, a range that encompasses the $7 \%$ value obtained from the simulation model in the previous section. The variance in the estimates of damping using the wavelet method is much higher than for the other methods. This can once again be attributed to the univariate and non-linear nature of the method which requires careful selection of an optimal signal for analysis. 


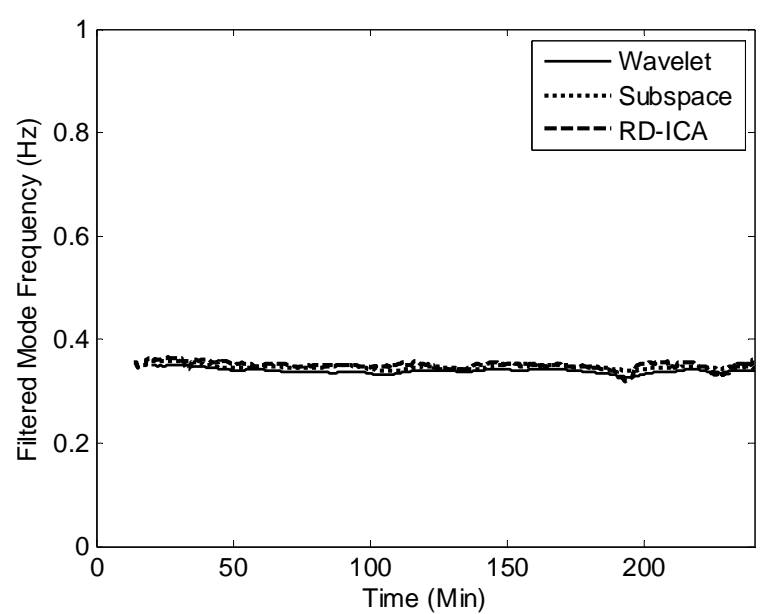

Fig. 12. Comparison of frequency estimates between the three methods. Input quantities are the measured bus voltage angle differences.

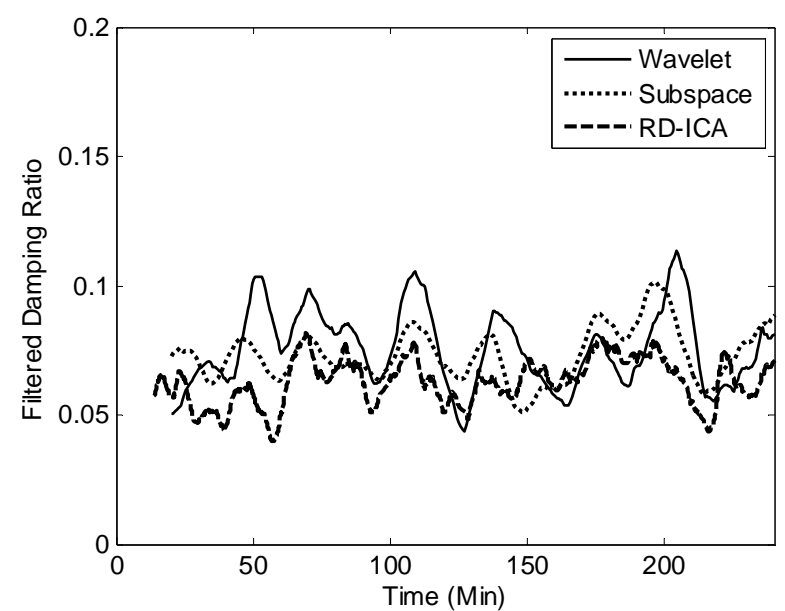

Fig. 13. Comparison of damping estimates between the three methods. Input quantities are the measured bus voltage angle differences.

\section{Multiple Mode Detection}

The main focus of this paper is on estimating the damping of the dominant $0.3 \mathrm{~Hz}$ inter-area mode because it sets the limit for power transmission from Finland to Sweden. However, it has been shown in [21], [23], and [27], for the subspace, RD-ICA and wavelet methods, that the methods are capable of simultaneously identifying multiple modes, including relatively closely-spaced modes. Modes are generally considered to be closely-spaced when the ratio between the frequencies of the modes is less than two, i.e. in the frequency spectrum they are within one octave.

Table 3 shows damping and frequency estimation results of the three methods for both the $0.3 \mathrm{~Hz}$ and $0.5 \mathrm{~Hz}$ modes of the simulated Nordic power system. The analyzed signals are in this case from Norway and Sweden, where especially the 0.5 $\mathrm{Hz}$ mode is observable. The results between the different methods agree quite well, indicating that the methods are capable of simultaneously identifying the closely spaced 0.3 $\mathrm{Hz}$ and $0.5 \mathrm{~Hz}$ modes in measurements from locations where both modes are observable. In the simulations, the same detailed model of the Nordic power system is used as in other parts of this paper.
Table 3. Multiple mode detection with simulated data. Time window length is $\mathbf{1 1}$ minutes, signal-to-noise ratio is $\mathbf{5}$ (linear scale).

\begin{tabular}{|l|l|l|l|}
\hline & \multicolumn{2}{l|}{$\begin{array}{l}\text { Frequency estimate with } \pm \text { one standard deviation } \\
\text { error bounds }\end{array}$} \\
\hline \hline & Subspace & RD-ICA & Wavelet \\
\hline \hline Frequency, 0.3 & $0.3104 \pm$ & $0.3140 \pm$ & $0.3106 \pm$ \\
Hz Mode & 0.0020 & 0.0089 & 0.0032 \\
\hline Damping, 0.3 & $0.0799 \pm$ & $0.0577 \pm$ & $0.0638 \pm$ \\
Hz Mode & 0.0063 & 0.0089 & 0.0055 \\
\hline Frequency, 0.5 & $0.5062 \pm$ & $0.5185 \pm$ & $0.5001 \pm$ \\
Hz Mode & 0.0015 & 0.0289 & 0.0010 \\
\hline Damping, 0.5 & $0.0472 \pm$ & $0.0584 \pm$ & $0.0579 \pm$ \\
Hz Mode & 0.0033 & 0.0108 & 0.0061 \\
\hline
\end{tabular}

\section{ASSUMPTION OF GAUSSIAN EXCITATION}

The RD-ICA and wavelet methods assume the excitation due to random loads during ambient operation is Gaussian. Whether the excitation is Gaussian or not depends on the time window of the data. If a major load switching event occurs within the window, or if diurnal variations in load are captured, then the distribution cannot be Gaussian. However, the methods presented in this paper use sliding windows from ambient operation of several minutes duration. On this time scale the load can be assumed to be stationary, and the accumulated load will be Gaussian distributed according to the central limit theorem [30]. To verify the Gaussian assumption a measurement of loads at a 400/110 kV substation in the Finnish grid was made and the result is shown in Fig. 14. As shown in the figure, this recording supports the assumption of a Gaussian distribution for the grid measurements used in Section VII B.

Further discussion of the Gaussian assumption is given in [31], where stochastic optimization is applied to express the demand distribution as a convex combination of the Gaussian mixture model. This means that the distribution is at any point a resultant Gaussian. Although the mean and variance is dependent on time, it always retains Gaussian equivalence. In addition, in a large power system the number of loads is usually very large and the individual loads are small compared to the system size.

Several other damping estimation methods also assume that the excitation has a Gaussian probability distribution function; see, e.g. [6], [32], [33]. The subspace method does not, however, carry this assumption and would therefore be a suitable method if there are concerns about non-Gaussian excitation. 


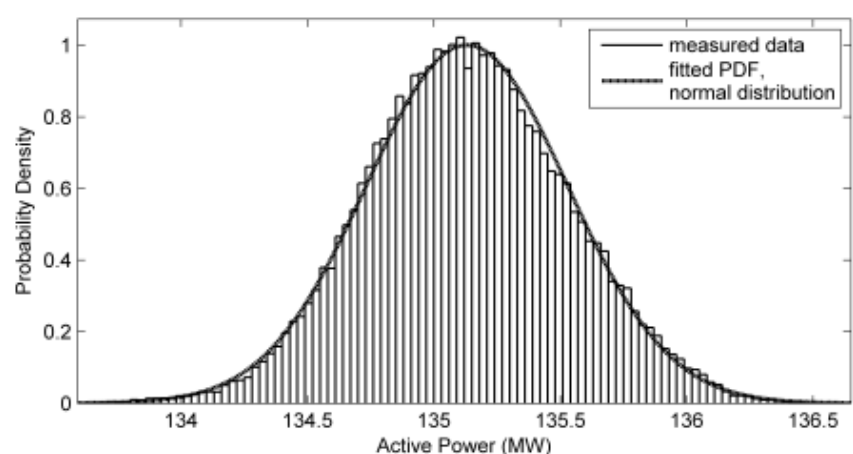

Fig. 14. Probability distribution of a Finnish urban load and the fitted normal distribution probability density function (PDF) of the measured 120 minutes period.

\section{CONCLUSIONS}

Given the consistency in the results presented in this paper, obtained using both simulated and measured data for the RDICA, subspace and wavelet methods, it can be concluded that the methods are all suitable for the estimation of frequency and damping of inter-area modes during ambient operation.

The frequency estimates are accurate and consistent for all methods. The damping results, on the other hand, are slightly lower than the reference value; the standard deviations of the estimates however account well for this difference. The methods produce consistent results with the selected measured and simulated quantities and regardless of the measurement noise. It has also been shown that an accurate estimate of the mode damping requires a data window length of at least 7 minutes in order to minimize the variance inherent to the methods. The methods all perform similarly when the energy of the mode is high in the signals but the RD-ICA and subspace methods have a lower variance when the mode energy is low. Multivariate analysis therefore has an advantage over univariate analysis by reducing the effect of a decrease in mode energy of one particular signal. The wavelet method is non-linear which also makes it sensitive to the averaging technique for obtaining a single damping estimate over a specific window, and hence this contributes to the higher variance in its results. Finally, the subspace method is expected to perform better than the RD-ICA and wavelet methods if the excitation in the system is non-Gaussian because the subspace method does not rely on a Gaussian assumption.

\section{Future WORK}

The scope of this paper is to compare three recently developed damping estimation methods, however the methodology applied here can be extended to compare the other methods in the damping estimation field too. An extensive comparison of all the available methods would facilitate the selection of a suitable method for each power system operator's specific purposes.

The Gaussian distribution of the power system excitation under the ambient conditions was shown to be true for the studied load and similar results have been reported in other studies. However, because several damping estimation methods rely on the Gaussian assumption, its validity is very important. An extensive study, beyond the scope of this paper, would help to further verify the assumption.

\section{ACKNOWLEDGMENT}

M. Laasonen, H. Kuisti, T. Kaukonen, M. Koskinen, K. Saarinen and J. Jyrinsalo from Fingrid Oyj are acknowledged for providing simulation models, measurement data, and industrial expertise. The authors wish to acknowledge financial support from European Commission through the Industry Academia Partnerships and Pathways project REALSMART.

\section{REFERENCES}

[1] J. Paserba (Convenor), Analysis and Control of Power System Oscillations: CIGRE Special Publication 38.01.07, 1996, vol. Technical Brochure 111.

[2] P. Pourbeik (Convenor), Wide-area Monitoring and Control for Transmission Capability Enhancement: CIGRE Working Group C4.601, 2007, vol. Technical Brochure 330.

[3] M. Zima, M. Larsson, P. Korba, Ch. Rehtanz, and G. Andersson, Design Aspects for Wide-Area Monitoring and Control Systems, Proceedings of the IEEE, Vo.93, No.5, May 2005, pp.980-996.

[4] D.J. Trudnowski and J.W. Pierre, "Overview of algorithms for estimating swing modes from measured responses," in Proc. 2008 IEEE Power Engineering Society General Meeting, 6 pp.

[5] R. Doraiswami, and W. Liu, "Real-time Estimation of the Parameters of Power System Small Signal Oscillations," IEEE Trans. Power Systems, vol. 8, pp. 74-83, Feb. 1993.

[6] M. G. Anderson, N. Zhou, J. W. Pierre, and R. W. Wies, "BootstrapBased Confidence Interval Estimates for Electromechanical Modes From Multiple Output Analysis of Measured Ambient Data," IEEE Trans. Power Systems, vol. 20, no. 2, pp. 943-950, May 2006.

[7] H. Ghasemi, C. A. Cañizares, and A. Moshref, "Oscillatory Stability Limit Prediction Using Stochastic Subspace Identification," IEEE Trans. Power Systems, vol. 21, no. 2, pp. 736-745, May 2007.

[8] M. Glickman, P. O'Shea, and G. Ledwich, "Estimation of Modal Damping in Power Networks," IEEE Trans. Power Systems, vol. 22, no. 3, pp. 1340-1350, Aug. 2007.

[9] R. A. Wiltshire, G. Ledwich, and P. O'Shea, "A Kalman Filtering Approach to Rapidly Detecting Modal Changes in Power Systems," IEEE Trans. Power Systems, vol. 22, no. 4, pp. 1698-1706, Nov. 2007.

[10] J. Y. Cai, Z. Huang, J. Hauer, and K. Martin, "Current Status and Experience of WAMS Implementation in North America," in Proc. 2005 IEEE/PES Transmission and Distribution Conference and Exhibition: Asia and Pacific, 7 pp.

[11] W. Sattinger, J. Bertsch, and P. Reinhardt, "Operational Experience with Wide Area Monitoring Systems," CIGRE 2006 Session, SC B5-216, Paris 27th Aug. - 1st Sep. 2006.

[12] A. B. Leirbukt, J. O. Gjerde, P. Korba, K. Uhlen, L. K. Vormedal, L. Warland, "Wide Area Monitoring Experiences in Norway," in Proc. 2006 IEEE PES PSCE, pp. 353-360.

[13] D. H. Wilson, "Wide-Area Measurement and Control for Dynamic Stability," in Proc. 2007 IEEE Power Eng. Soc. General Meeting, 5 pp.

[14] C.-L. Su, and B.-Y. Jau, "Visualization of Wide Area Dynamics in Power Network for Oscillatory Stability Assessment," in Proc. 2007 Int. Conf. on Intelligent Systems Applications to Power Systems, 6 pp.

[15] G. Ledwich, D. Geddey, and P. O'Shea, "Phasor Measurement Unit's for system diagnosis and load identification in Australia," in Proc. 2008 IEEE Power Engineering Society General Meeting, 6 pp.

[16] J. Turunen, M. Larsson, P. Korba, J. Jyrinsalo, and L. Haarla, "Experiences and Future Plans in Monitoring the Inter-area Power Oscillation Damping," in Proc. 2008 IEEE Power Engineering Society General Meeting, 8 pp.

[17] K. P. Poon, and K. C. Lee, "Analysis of Transient Stability Swings in Large Interconnected Power Systems by Fourier Transformation," IEEE Trans. Power Systems, vol. 3, no. 4, pp. 1573-1581, Nov. 1988.

[18] J. J. Sanchez-Gasca, and J. H. Chow, "Performance Comparison of Three Identification Methods for the Analysis of Electromechanical 
Oscillations," IEEE Trans. Power Systems, vol. 14, no. 3, pp. 995-1002, Aug. 1999.

[19] D. Ruiz-Vega, A. R. Messina, and M. Pavella, "On-Line Assessment and Control of Transient Oscillations Damping," IEEE Trans. Power Systems, vol. 19, no. 2, pp. 1038-1047, May 2004.

[20] A. R. Messina, and V. Vittal, "Nonlinear, Non-Stationary Analysis of Interarea Oscillations via Hilbert Spectral Analysis," IEEE Trans. Power Systems, vol. 21, no. 3, pp. 1234-1241, Aug 2006.

[21] M. Larsson, and D.S. Laila, "Monitoring of inter-area oscillations under ambient conditions using subspace identification," Proc. 2009 IEEE Power Engineering Society General Meeting, 6 pp.

[22] P. Overschee and B. de Moor, Subspace Identification for Linear Systems: Theory - Implementation - Applications. Kluwer Academic Publishers, 1996.

[23] J. Thambirajah, N. F. Thornhill, and B. C. Pal, "A Multivariate Approach Towards Inter-Area Oscillation Damping Estimation Under Ambient Conditions Via Independent Component Analysis And Random Decrement," IEEE Transactions on Power Systems, 2010, Accepted for publication.

[24] C. Xia, and J. Howell, "Isolating Multiple Sources of Plant-Wide Oscillations via Independent Component Analysis," Control Engineering Practice, 13(8), 2004, pp. 1027-1035.

[25] A.H. Cole, "Failure Detection of a Space Shuttle Wing Flutter by Random Decrement," NASA, TMX-62, 041, 1971.

[26] J. Turunen, T. Rauhala, and L. Haarla, "Selecting Wavelets for Damping Estimation of Ambient-excited Electromechanical Oscillations", IEEE Power \& Energy Society General Meeting. 2010.

[27] J. Turunen, L. Haarla, and T. Rauhala, "Performance of Wavelet-Based Damping Estimation Method under Ambient Conditions of the Power System," 2010 IREP Symposium.

[28] I. Daubechies, Ten Lectures on Wavelets, Philadelphia, PA: SIAM, 1992, pp. 1-7.

[29] S. Elenius, K. Uhlen, and E. Lakervi, "Effects of Controlled Shunt and Series Compensation on Damping in the Nordel System," IEEE Trans. Power Systems, vol. 20, no. 4, pp. 1946-1957, Nov. 2005.

[30] M. Blum, "An Alternate Proof of the Central Limit Theorem for Sums of Independent Processes," Proceedings of the IEEE, vol. 54, no. 6, pp. 878-879, 1966.

[31] R. Singh, B. C. Pal, and R. A. Jabr, "Statistical Representation of Distribution System Loads Using Gaussian Mixture Model," IEEE Trans. Power Systems, vol. 25, no. 1, pp. 29-37, Feb. 2010.

[32] D. J. Trudnowski, J. W. Pierre, N. Zhou, J. F. Hauer, and M. Parashar, "Performance of Three Mode-Meter Block-Processing Algorithms for Automated Dynamic Stability Assessment," IEEE Trans. Power Systems, vol. 23, no. 2, pp. 680-690, May 2008

[33] G. Liu and V. Venkatasubramanian, "Oscillation Monitoring from Ambient PMU Measurements by Frequency Domain Decomposition," IEEE International Symposium on Circuits and Systems (ISCAS). pp. 2821-2824, 2008

\section{BIOGRAPHIES}

Jukka Turunen (S'07) is currently a research scientist in Aalto University School of Electrical Engineering, Finland.

Jegatheeswaran Thambirajah (S'06) is working towards a Ph.D degree in Imperial College London, U.K.

Mats Larsson (M'01) is working on the research and development of widearea stability controls at ABB Corporate Research, Switzerland.

Bikash C. Pal (M'00-SM'02) is currently a Reader in the Department of Electrical and Electronic Engineering, Imperial College London.

Nina Thornhill (SM'93) is currently a Professor in the Department of Chemical Engineering at Imperial College London where she occupies the ABB/RAEng Chair of Process Automation.

Liisa Haarla (M'05, SM'10) is a Professor of power transmission systems in Aalto University School of Electrical Engineering, Finland.

William W. Hung is currently working as a technical specialist at National Grid Plc, UK.

Alex M. Carter is currently working as a transmission issues manager at National Grid Plc, UK.
Tuomas Rauhala (S'04, M'08) is currently working as a specialist at Fingrid Oyj. He is also a Ph.D. student in Tampere University of Technology. 